\title{
Trivium
}

Revue franco-allemande de sciences humaines et sociales - Deutsch-französische Zeitschrift für Geistesund Sozialwissenschaften

15 | 2013

La science pense en plusieurs langues

\section{»Lost in translation«. Übersetzung und kultureller Dialog}

Bernd Stiegler

URL : http://journals.openedition.org/trivium/4559

DOI : $10.4000 /$ trivium.4559

ISSN : $1963-1820$

Éditeur

Les éditions de la Maison des sciences de l'Homme

\section{Référence électronique}

Bernd Stiegler, « »Lost in translation«. Übersetzung und kultureller Dialog », Trivium [Online], 15 | 2013, online erschienen am 09 Dezember 2013, abgerufen am 08 September 2020. URL : http:// journals.openedition.org/trivium/4559; DOI : https://doi.org/10.4000/trivium.4559

Ce document a été généré automatiquement le 8 septembre 2020

\section{(c) $(1) \Theta \Theta$}

Les contenus des la revue Trivium sont mis à disposition selon les termes de la Licence Creative Commons Attribution - Pas d'Utilisation Commerciale - Pas de Modification 4.0 International. 


\title{
»Lost in translation«. Übersetzung und kultureller Dialog
}

\author{
Bernd Stiegler
}

1 »Lost in translation«, der aktuelle Film von Sofia Coppola, der Tochter des amerikanischen Regisseurs Francis Ford Coppola, ist ein großer Erfolg an den Kinokassen und bei den Kritikern. Dieser Erfolg erklärt sich nicht zuletzt durch die überzeugende Darstellung einer Fremdheit, die in diesem Film vordergründig als Konfrontation mit einer anderen Kultur dargestellt wird, die aber mit der Erfahrung einer Fremdheit in den unmittelbaren persönlichen Beziehungen und einer Fremdheit zu sich selbst einhergeht. Die Fremdheit einer anderen Kultur, in diesem Fall nun Japans, die hier in überaus plakativer, ja klischeehafter Form in mittlerweile schon klassisch fremden Bildern vermittelt wird, ist Spiegel der Erfahrung einer Fremdheit, die sich in der vertrauten Nähe eingenistet hat. Und das Fremde, das wir sehen, ist uns auf eine eigentümliche Weise bereits vertraut. Vertraut durch eine lange Tradition des kulturell tradierten Fremden, auf das jede Darstellung des Fremden zurückgreifen kann. Wenn die Fremdheit Japans dargestellt werden soll, so ist es ausgemachte Sache, daß Neonreklamen mit japanischen Schriftzeichen eine ebenso prominente Rolle einnehmen werden wie Karaoke-Bars und Zen-Tempel. Sämtliche Formen dieses inszenierten fremden Blicks sind langerprobt und vielfach bewährt. Sie sind Teil einer cineastischen wie literarischen Ikonologie, die auf ein setting von Bildern zurückgreifen kann. In der Tradition der Literatur- und Kulturwissenschaft ist dieser Zusammenhang unter dem Label der "Imagologie« breit erforscht. ${ }^{1}$ Und auch Übersetzungen sind Teil dieses Programms einer inszenierten Fremdheit, die ihre Verankerung in der eigenen Kultur hat und letztendlich mehr über diese als über die fremde Kultur aussagt - oder allenfalls über das festgefügte, opake und letztlich wahrnehmungsresistente Bild einer anderen Kultur. Ähnlich fällt ja auch die conclusio der imagologischen Forschung aus, die ein wenig frustriert, aber gleichwohl beobachtungssicher die Langelebigkeit (in der Regel) deutscher Vorurteile über Frankreich und französischer Vorurteile über Deutschland konstatiert, um dann auf die neuen Bilder zu setzen, die der europäische Gedanke ins Spiel bringen könnte. Dann würde allerdings das alte Spiel mit neuen Karten fortgesetzt und an der Stelle von 
Frankreich oder Deutschland stünden nun die Vereinigten Staaten ${ }^{2}$, afrikanische Länder oder eben Japan ...

2 Ähnlich verhält es sich auch mit dem Film "Lost in translation", der nur deshalb einen Platz auf den Nominierungslisten für den Oscar erhalten hat, weil die Darstellung bereits Teil der amerikanischen Vorstellung des Fremden ist. "Lost in translation« ist jedoch kein Film über Japan und letztendlich auch kein Film über das amerikanische Japan-Bild, sondern ein Film über eine Beziehung zwischen einem verheirateten Familienvater mittleren Alters und einer jungen verheirateten kinderlosen Frau, deren Parameter maßgeblich durch die amerikanische (und vielleicht auch kontinentale) Kultur gegeben sind. Wir bewegen uns auf dem sicheren Boden einer westlichen Sicht der Dinge. Keine Übersetzung eines japanischen Romans würde daran etwas ändern, im Gegenteil: die Übersetzung wäre Teil dieser Strategie, Fremdheit zu inszenieren und allmählich in die eigene Kultur zu überführen. Auch für das Fremde ist hier ein Ort vorgesehen. In der Geschichte der Literatur und ihrer Übersetzungen wären eine Reihe von Beispielen anzuführen, die von den Klassikern der ostasiatischen Philosophie über Übersetzungen von mythischen Erzählungen fremder Völker und Länder bis hin zu den »Erzählungen aus tausendundeiner Nacht « im Kontext des Orientialismus ${ }^{3}$ reicht. In der Literatur- und Kulturwissenschaft nehmen die Colonial und Postcolonial Studies ihren Ausgang von ähnlichen Phänomenen und untersuchen ihrerseits den kulturell und historisch codierten Blick auf das Fremde, der Fremdheit immer nur als Teil des Eigenen begreift. ${ }^{4}$

In "Lost in translation" ist uns das Fremde als eine Form der Fremdheit vertraut, die wir schon lange kennen und die ihrerseits, wenn man genau hinsieht, so festgelegt und zugleich so kontingent ist, daß sie keine Übersetzung duldet, da das, was zu übersetzen wäre, nichts als nur banal wäre: Straßenschilder, Leuchtreklamen, soziale Konventionen und ostasiatische Formen der Freizeitgestaltung. Und die Evidenz der Fremdheit ist ebenso stark wie das Wissen um die Bedeutung des vermeintlich kulturell Entrückten. Die Fremdheit ist hier eine Inszenierung, die bei aller und trotz aller Übersetzung nichts als die eigene Kultur im Blick hat. Dieses erste Modell der Fremdheit und auch der Übersetzung können wir als Projektion der eigenen Fremdheit bezeichnen. Es ist eine vertraute Fremdheit.

4 Demgegenüber träumt Roland Barthes in seinem mittlerweile fast klassischen Text L'empire des signes von 1970 noch von einer Welt, an der unsere Vertrautheit zerschellt, von einer unübersetzbaren Sprache, von einem Reich der Zeichen, das nur eines möglich macht: "descendre dans l'intraduisible«. ${ }^{5}$ Roland Barthes begründet diesen Traum mit dem Wunsch nach dem Entkommen aus den Zwängen der abendländischen Kultur, die nicht zuletzt Zwänge der Begriffe sind, die uns, so unterstreicht es insbesondere die philosophische Theorie seit Heidegger, seit der griechischen Antike überliefert sind und unsere Vorstellungen bestimmen. Japan, diese fremde Kultur, soll uns bei Barthes unserer eigenen Kultur gegenüber in ein Verhältnis der Fremdheit setzen, uns der "clôture e einer »époque historico-métaphysique «", wie es dann bei Derrida heißt, entrücken. Auch Foucault und selbst Heidegger haben Reisen nach Japan unternommen und von beiden Reisen sind Texte überliefert, die uns heute als eigentümliche, ein wenig erratische Dokumente erscheinen, in denen die seltsame Verbindung der eigentümlichen Beziehung zwischen dem Nahen, ja dem Nächsten und dem Fernen, ja dem Fernsten eine entscheidende Rolle spielt. So fühlte sich etwa Heidegger in Japan am besten verstanden ${ }^{7}$; Japan war für ihn ein Ort und eine 
Tradition, in der er die Einklammerung der eigenen Tradition, wie er sie in Europa versucht hatte, in praxi vorgeführt bekam. ${ }^{8}$ Diese Anerkennung des Fremden läuft durchweg Gefahr, wieder in eine bloße Projektion des Eigenen zurückzufallen, in eine "narzißtische« Projektion der eigenen Wünsche und des eigenen Wunschbildes seiner selbst, und sei es nur das der eigenen Theorie.

Diese zweite Form der Fremdheit wäre als Utopie des Unübersetzbaren zu bezeichnen, als Produktion einer Distanz zu unserer eigenen Kultur, die ihre Vertrautheit aber partout nicht verlieren will. Daher werden in der philosophischen Theorie eine Reihe von Verfahren, Theorien oder Deutungsperspektiven entworfen, die genau diese Frage in den Blick nehmen und versuchen, genau diese Distanz herzustellen. Eines dieser Verfahren ist etwa die Theorie (und philologische, philosophische und mitunter auch performative Praxis) der Destruktion, bei der es auch immer um Fragen der Übersetzung geht. Die gesamte Theorie und Praxis der europäischen Avantgarde, vom Futurismus und dem Dadaismus über den Surrealismus und das Bauhaus bis hin zu Theoretikern wie Ernst Jünger und Walter Benjamin ${ }^{9}$ ist maßgeblich durch eine Poetik der Destruktion bestimmt, die auch in vielfältiger Weise Bezüge zur Übersetzung aufweist. Und wenn etwa Martin Heidegger seinerseits die Praxis der Lektüre als dezidierte Destruktion der abendländischen Metaphysik begreift und auch seine »Rückkehr« zu den Vorsokratikern vor allem darauf zielt, hinter die Setzungen der abendländischen Metaphysik zu gelangen, so hat dies ebenfalls Konsequenzen für die Übersetzung und ihre Theorie. Dies zeigt sich sowohl an Heideggers betontem Eingehen auf Fragen der Etymologie und seiner spezifischen Deutungspraxis (wie sie sich in seinen Übersetzungen griechischer Begriffe, wie seine berühmte Deutung des Wortes "aleitheia", ebenso zeigt wie in seinen späten Texten, in denen immer wieder etymologische Deutungen eine zentrale philosophische Bedeutung erhalten) als auch in seinen eigenen Übersetzungen klassischer Texte und Begriffe, die einen Abstand zu der eigenen Tradition suchen und diesen auch auszuloten versuchen.

Ähnliches gilt auf französischer Seite auch für Jacques Derrida, dessen Werk eine Vielzahl von Bezügen zur Theorie der Übersetzung aufweist und die philosophische Nähe zu Martin Heidegger nicht verleugnet ${ }^{10}$, aber auch für Literaten wie Raymond Queneau, die Surrealisten oder Henri Michaux (der, wie Queneau, einige Jahre lang den Surrealisten ja auch nahestand) und Theoretiker wie Michel Foucault und weite Teile der Gruppe Tel Quel, um nur einige wenige Beispiele anzuführen. Viele weitere Namen wären hier anzuführen - auch aus der Literatur und Theorie jenseits des Rheins.

7 Während etwa Queneau die sogenannten »fous litteraires« in seinem Roman Les enfants du limon ${ }^{11}$ der abendländischen Tradition gegenüberstellt und auch Foucault später (was L'histoire de la folie à l'âge classique ${ }^{12}$ ebenso zeigt wie mehrere Aufsätze ${ }^{13}$ ) den Wahnsinn als fremde Stimme innerhalb der eigenen Kultur versteht und sich seinerseits für die »fous litteraires« begeistert, so findet sich bei Michaux eine komplexe Reflexion über die Erfahrung der Drogen, die ihrerseits die kulturellen Vorgaben der "écriture « des Schriftstellers einzuklammern sucht. ${ }^{14}$ Die Surrealisten haben eine Vielzahl von Verfahren entwickelt, die eigene Kultur für eine kurze Zeit außer Kraft zu setzen, von denen die "écriture automatique« nur das bekannteste ist, und auch die Gruppe Oulipo, der neben Queneau auch Perec, Calvino oder Roubaud angehörte, hat ähnliches versucht - nun aber mit strengen formalen Vorgaben. ${ }^{15}$ Für alle gilt, daß die Fremdheit ein Distanzierungsversuch von der eigenen Kultur darstellt und in keiner Weise auf diese reduzierbar ist. Diese Fremdheit ist der Traum einer 
anderen Kultur mit einer anderen Geschichte, einer anderen Sprache und Tradition, einer anderen Bildlichkeit und einem anderen Denken.

Während beim ersten Modell der Fremdheit und auch der Übersetzung die Fremdheit vor allem Projektion der eigenen Fremdheit war, für sie aber zumindest ein Platz im Herzen der eigenen Kultur vorgesehen war, so ist das zweite Modell fraglos radikaler, aber zugleich ebenso aporetisch. Hier ist die Fremdheit Gegenmodell der eigenen Kultur und zwar der eigenen Kultur als solcher. Dementsprechend ist die Übersetzung ein heikles Unterfangen, da per se kontraproduktiv, es sei denn innerhalb eines theoretischen Entwurfs, in dem ihr ein Platz zugebilligt wird, da die Theorie auf etwas Vergleichbares zielt. Dieses Modell radikalisiert die Erfahrung der Fremdheit und macht aus ihr eine Alternative zu der eigenen historisch wie kulturell codierten und als bedrängend erfahrenen Tradition. Hier geht es nicht um ein Spiegelbild des Eigenen, sondern um sein Gegenbild, um ein Gegenmodell der eigenen Kultur - mit all den Schwierigkeiten, die dies mit sich bringt. Es geht letztlich um die Übersetzung und/ oder die Fremdheit als Utopie.

9 Zwischen diesen beiden Positionen - der Projektion und der Utopie - steht meines Erachtens das Modell der Übersetzung. Ich möchte hier die These vertreten, daß die Übersetzung weniger einer kulturellen Verständigung dient, sondern viel mehr dazu, eine spezifische Form der Fremdheit zu erzeugen, die, wenn die Übersetzung gelingt, in beiden Kulturen Fremdheitseffekte hervorruft, die dann ihrerseits zu einer doppelten und zugleich wechselseitigen kulturellen Verständigung führen. Sie ist weder vertraute Fremdheit des inszenierten kulturellen Fremden, noch Utopie einer Fremdheit, die als Negativ des eigenen kulturellen Horizonts fungieren würde.

Dies sei am Beispiel der deutsch-französischen Übersetzungen im Bereich der Philosophie im 20. Jahrhundert kurz erläutert. Wenn zu Beginn des 20. Jahrhunderts, wie Vincent Descombes dies nachgezeichnet hat ${ }^{16}$, neben Nietzsche die drei großen »Hs« Hegel, Husserl, Heidegger für die Hauptpositionen der französischen Philosophie von Sartre über Foucault und Levinas bis hin zu Derrida maßgebliche Bedeutung haben, so führt dies $\mathrm{zu}$ philosophischen Positionen, die nicht nur mit der deutschen Philosophie wenig, ja wahrscheinlich gar nichts zu tun haben, sondern sich auch deutlich von deutschen Lesarten von Hegel, Husserl und Heidegger unterscheiden. Man mag einwenden, daß das, was deutsche Literaturtheoretiker in den 70er Jahren (ich denke hier insbesondere an Jauß und Iser) und einige wenige Philosophen, wie etwa Hans Blumenberg, vorgelegt haben, als »deutsches Pendant« zur französischen postoder neostrukturalistischen Theorie angesehen werden kann. Aber dies würde - nur ein wenig "phasenverschoben « - den Befund bestätigen, daß die Übersetzung in der Tat Fremdheitseffekte hervorruft, da die Wissenschaftsfelder gänzlich andere sind (die Philosophie wandert in die Literaturtheorie) und auch die Position Blumenbergs nicht zuletzt aufgrund seiner $»$ metaphorologischen $«{ }^{17}$ Ausrichtung in der Philosophie seiner Zeit ohne großen Widerhall blieb. So ist es auch nicht erstaunlich, daß gerade solche Philosophen oder Theoretiker, die sich um den kulturellen wie theoretischen Austausch verdient gemacht haben, in dem jeweils anderen Land erhebliche Probleme haben, als maßgebliche und wichtige Theoretiker wahrgenommen zu werden. Ihr spezifischer Beitrag zur Debatte ist aufgrund der neuen Verortung in einem diskursiven theoretischen Zusammenhang von der Ausgangsposition nun so weit entfernt, daß die Rückübersetzung der Theorie erhebliche Schwierigkeiten macht und die Theorien, die am Anfang standen, für die Ausgangsländer kaum noch zu erkennen 
sind. Die "Übersetzung" einer Theorie in einen anderen kulturellen oder theoretischen Kontext erfordert bestimmte Akzentuierungen, die von den vermeintlich selbstverständlichen Deutungen der Ausgangstheorie weit entfernt sind und diese für die Rezeption im eigenen Land verstellen. Dies gilt etwa, um nur zwei Beispiele anzuführen, für Manfred Frank, ohne den in Deutschland eine Rezeption des Post- oder Neostrukturalismus deutlich andere Konturen gewonnen hätte ${ }^{18}$, bei dem die Übersetzungen seiner Arbeiten in Frankreich aber kaum Resonanz fanden (und kein Franzose würde Derrida mit Foucault, Barthes oder Levi-Strauss in einer gemeinsamen Traditionslinie verorten oder gar unter einem gemeinsamen theoretischen »Label« vereinen), wie auch umgekehrt - für Rainer Rochlitz, der sich um das Werk von Jürgen Habermas und der Frankfurter Schule in Frankreich verdient gemacht hat wie kein zweiter, zugleich aber auch mit seinen eigenen Werken in Deutschland wenig Erfolg hatte, oder für Jacques Bouveresse, der in Frankreich maßgeblich zur Entdeckung Wittgensteins beigetragen hat und eine durchaus eigenständige Stimme in der analytischen Philosophie geworden, in Deutschland aber nach wie vor gänzlich unbekannt ist. Diese Liste wäre fortzusetzen und auch auf historische Beispiele zu übertragen. Allen Beispielen ist gemeinsam, daß die Übersetzungsversuche - und das ist hier im weiteren Sinne zu verstehen - notwendigerweise neue Kontextualisierungen vornehmen müssen, durch die die Ausgangstexte oder -theorien in spezifischer Weise re- wie dekontextualisiert werden. Dies zieht zweifache Fremdheitseffekte nach sich: Auf der einen Seite erscheint plötzlich in einer theoretischen Tradition eine neue Stimme, die allerdings ohne Übersetzungsversuch und ohne Rezeption stumm und ungehört bliebe; auf der anderen führt diese Neu- und Umformulierung dazu, daß diese die Theorie für das Ausgangsland mitunter bis zur Unkenntlichkeit entstellt. Eine durchaus paradoxe Situation, aber keineswegs der Endpunkt der Übersetzungsbemühungen und -erfolge, wie es einige Beispiele zeigen, die einen weiteren zeitlichen Horizont ansetzen.

11 Alexandre Kojeves Vorlesungen über Hegel etwa ${ }^{19}$, die als Vermittler für die französische Hegel-Rezeption eine herausragende Bedeutung haben, sind mit dem deutschen Hegelianismus dieser Zeit nicht zu versöhnen, und Jacques Derridas Übersetzung und Deutung von Husserls Ursprung der Geometrie hat mit einer Phänomenologie deutscher Provenienz ebenso wenig zu tun. Und umgekehrt ist die Situation kaum anders: Hegel, Husserl und Heidegger entsprechen keiner der bekannten Positionen und führen in die französische philosophische Szene eine Stimme ein, die fremd ist und aus dieser Fremdheit ihre Effekte bezieht. (Die Bedeutung, die diese neue Stimme haben kann, hat nun eigentümlicherweise zwar mit der Existenz von Übersetzungen, nicht aber notwendigerweise mit ihrer Qualität etwas zu tun. So gab es etwa eine intensive Benjamin-Rezeption in Frankreich trotz einer Übersetzung von Maurice de Gandillac, die mit dem deutschen Text recht wenig zu tun hatte. ${ }^{20}$ )

Nun findet zwei Jahrzehnte später in Deutschland eine Gegenbewegung statt, indem jetzt die französische Gegenwartsphilosophie ihrerseits breit rezipiert wird. Auch hier entsprechen den Positionen Derridas, Levinas', Barthes' oder Foucaults keine bekannten theoretischen Ansätze - weder die der ersten oder zweiten Frankfurter Schule noch der deutsche Existentialismus oder die Gruppe "Poetik und Hermeneutik" und die analytische Philosophie wittgensteinscher Prägung, um nur einige wenige Beispiele zu nennen. Und auch hierzulande nehmen die Positionen, die in Frankreich deutlich voneinander geschieden werden (wer je ein Seminar über Foucault in 
Frankreich besucht und dabei irgendweise annimmt, es würde dort auch über Derrida und Barthes oder Lacan gesprochen, wie dies in Deutschland selbstverständlich der Fall wäre, weiß wovon ich spreche), eine neue Gestalt an und sollen plötzlich miteinander verwandt sein. Vom Neostrukturalismus über den Poststrukturalismus bis hin zur Postmoderne werden immer neue Labels gefunden, die die Theorien vereinen sollen und derer sich die Theoretiker kaum erwehren können. Gleichwohl setzt etwa ein Jahrzehnt später eine Diskussion ein, die nun die Frankfurter Schule mit Foucaults Machttheorie entspinnt oder Derrida mit Benjamin und Benjamin mit Derrida liest. Es ergibt sich ein eigentümlicher Dialog, der Nähe wie Ferne gleichermaßen einschließt und dies auf beiden Seiten der Übersetzung.

Nietzsche aus Frankreich, so lautet der Titel eines exemplarischen Bandes, der eben einen anderen Nietzsche präsentiert - aber mit einer Stimme die ihrerseits der deutschen Rezeption und dann auch der italienischen, die dann immerhin zur Edition der Sämtlichen Schriften durch Colli/Montinari führte, ein Timbre verleiht, das ihr vorher fehlte - eine Rauheit der Stimme, die den bekannten Tonfall verändert, ohne das Vertraute ganz zu nehmen.

\section{NOTES}

1. Vgl. etwa die einschlägigen Publikationen von H. Dyserinck, W. Leiner oder M. S. Fischer.

2. Auch hier liegen eine Reihe von Publikationen bereits vor. Vgl. expl. Claus Offe, Selbstbetrachtung aus der Feme. Amerikareisen von Tocqueville, Weber und Adorno, Frankfurt/M. 2004.

3. Vgl. exemplarisch und materialreich Edward Said, Orientalisme London 1978, das mittlerweile zum Standardwerk geworden ist. Vgl. auch J. H. Mc Kenzie, Orientalism. History, Theory, and the Arts, Manchester 1995.

4. Vgl. die Übersicht in: B. Moore-Gilbert, Postcolonial Theory. Contexts, Practices, Politics, London 1997; und P. Childs und P. Williams, An Introduction to Post-Colonial Theory, London 1997.

5. Roland Barthes, L'empire des signes, in: ders., Euvres complètes, hrsg. von Éric Marty, Bd. 2,19661973, Paris 1994, S. 742-831, S. 748. Zu Barthes Text vgl. Antje Landmann, "Zeichenleere«. Roland Barthes interkultureller Dialog mit Japan, München 2003.

6. Vgl. Jacques Derrida, De la grammatologie, Paris 1967, S. 14.

7. Vgl. exempl. H. Buchner (Hrsg.): Japan und Heidegger, Sigmaringen 1989.

8. Vgl. exempl. Michel Foucault, Michel Foucault und das Zen: ein Aufenthalt in einem ZenTempel, in: Dits et Ecrits, Band III, Frankfurt/M. 2003, S. 776-782.

9. Vgl. hierzu expl. Bernd Stiegler, La destruction et l'origine: Ernst Jünger et Walter Benjamin, in: Littérature, Nr. 112, Dezember 1998, S. 112-126.

10. Zu beiden Punkten: vgl. De l'esprit, Paris 1986; dt. Übersetzung: Vom Geist. Heidegger und die Frage, übersetzt von Alexander García Düttmann, Frankfurt/M. 1988.

11. Raymond Queneau, Les enfants du limon, Paris 1938.

12. Michel Foucault, L'histoire de la folie à l'âge classique, Paris 1961; dt. Übers. Wahnsinn und Gesellschaft. Eine Geschichte des Wahns im Zeitalter der Vernunft, übers. von Ulrich Köppen, Frankfurt/M. 1969.

13. Vgl. Michel Foucault, Sieben Thesen über den siebten Engel, in: ders., Dits et Ecrits. Schriften, Bd. 2, Frankfurt/M. 2002, S. 17-32. 
14. Das ist ein Topos der Drogenliteratur - zumindest des 20. Jahrhunderts.

15. Vgl. die Textsammlungen: Oulipo. La Bibliothèque Oulipienne, Paris $1987 \mathrm{ff}$. In diesen Bänden sind eine Vielzahl von kleineren, verstreut und in der Regel entlegen erschienenen Einzelpublikationen versammelt.

16. Vincent Descombes, Das Selbe und das Andere. Fünfundvierzig Jahre Philosophie in Frankreich, 1933-1978, übers. von Ulrich Raulff, Frankfurt/M. 1981.

17. Vgl. expl. Hans Blumenberg, Paradigmen zu einer Metaphorologie, Frankfurt/M. 1997; sowie ders., Ästhetische und metaphorologische Schriften, hrsg. von Anselm Haverkamp, Frankfurt/M. 2002.

18. Vgl. Manfred Frank, Was ist Neostrukturalismus?, Frankfurt/M. 1983, ein Buch, das seinerzeit die Entdeckung der französischen Gegenwartsphilosophie maßgeblich beförderte. Gleichwohl ist das Werk Manfred Franks keineswegs auf diese Auseinandersetzung oder gar diesen Theorieimport beschränkt; er ist fraglos einer der großen internationalen Spezialisten für die Frühromantikforschung und die Theorie des Selbstbewußtseins und hätte daher mit Fug und Recht in Frankreich auch als Theoretiker »entdeckt « werden können.

19. Alexandre Kojève, Hegel. Eine Vergegenwärtigung seines Denkens. Kommentar zur» Phänomenologie des Geistes«, übers. von Iring Fetcher und Gerhard Lehmbruch, Frankfurt/M. 1975; eine erweiterte Neuausgabe erscheint 2004.

20. Eine Stichprobe seiner Übersetzung der Aufsätze ergab, daß Teile der Texte fehlen, andere wiederum nur eine Improvisation nach einem Thema von Walter Benjamin darstellen. Inzwischen hat allerdings Gallimard eine sehr sorgfàltig editierte und genau übersetzte Ausgabe vorgelegt, die die über Jahrzehnte bestimmende von de Gandillac ablöst. Ähnliche Beispiele wären anzuführen - diesseits und jenseits des Rheins.

\section{INDEX}

Mots-clés : traduction, étrangeté, réception, contextualisation

Schlüsselwörter : Übersetzung, Fremdheit, Rezeption, Kontextualisierung

\section{AUTEUR}

\section{BERND STIEGLER}

Bernd Stiegler ist Professor für Neuere deutsche Literatur an der Universität Konstanz. Nähere Informationen finden Sie hier. 